\title{
Finite Element Analysis of the Continuous Dynamic ECAE Forming Process of Al-Zr Resists Heat Alloy
}

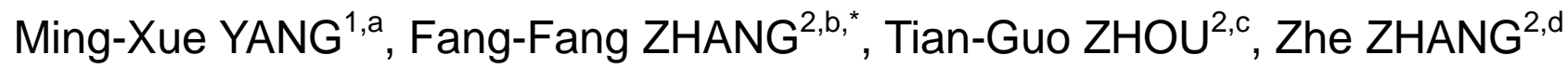 \\ ${ }^{1}$ College of Information Engineering, Shenyang University, Shenyang, Liaoning, China \\ ${ }^{2}$ College of Mechanical Engineering, Shenyang University, Shenyang, Liaoning, China \\ aymxIn@163.com, bzhangff90@163.com, czhoutg64@163.com, d995423221@qq.com \\ * Corresponding author
}

Keywords: Al-Zr Alloy, Finite Element Method, Continuous ECAE Forming, Deform-3D.

\begin{abstract}
The continuous ECAE forming process of $\mathrm{Al}-\mathrm{Zr}$ resistant heat alloy was simulated by finite element method with DEFORM-3D. The rigid viscoplastic finite element model of continuous ECAE forming process was established. The feasibility of continuous ECAE forming process was verified. The effective stress and strain and the temperature field distribution of the continuous ECAE forming process were analyzed. The results show that the ECAE forming process can be continuous with the reasonable die structure and process parameters. The severe deformation mainly occurred in the corner zone of the ECAE die in continuous ECAE forming process, the maximum value of temperature appears in the entrance of the ECAE die, the results are of very useful for practical production.
\end{abstract}

\section{Introduction}

Because traditional ECAE process cannot produce continuously, the samples prepared by that is too small to be satisfied for the requirement of industry and customer, the use of them are limited. Over the past 10 years, the question has been widely studied in the world [1-6]., a continuous shearing process (Conshearing) was proposed by Saito in 2002, the forming theory is the materials driven by the friction produced by the rotation working roll or the transfer, the sample continuous filled the die cavity and move to the cavity exit, the approximate ideal shear deformation was found in the junction of the two channels, achieved a continuous equal channel angular extrusion, and the aluminum alloy strip can be produced successfully [7]. The results show that the grains can be effectively refined by continuous ECAE and shear deformation textures obtained with properties improved. After that the aging properties of AL-Mg-Si alloy prepared by dynamic aging ECAE process (DAECAE) were studied by M. Cai Hans and his college in 2004. The results show that DAECAE process has the advantages of refining the Al-Mg-Si alloy grain size, promoting the strengthening phase element $\mathrm{Mg} \backslash \mathrm{Si}$ precipitated from the $\mathrm{Al}$ matrix rapidly or completely and their corresponding dynamic nucleation and nodularization of precipitated phase occurred, so the strength and elongation of the alloy can be greatly improved.

Continuous ECAE technology can be not only used in the large-scale industrial production of ultrafine grained materials, but also used in dynamic aging heat-treatment process. Fine grain strengthening combined with dynamic effects, can improve the comprehensive performance of the material. This technology can make constructional materials and also can be used to make functional materials, including conductive materials. It is of important for future research $[8,9]$.

Our group designed continuous ECAE aging forming equipment, the power of which is provided by the friction of the speed difference between the workpiece and tools. By the combination of continuous ECAE forming and dynamic aging forming, high strength and high conductivity aluminum alloy wire is expected to produce, a new method to produce high performance aluminum wire can be developed. Its principle diagram was shown in Fig.1. The whole system is made up of ECAE punch A, ECAE punch B, guide wheel and ECAE concave die. The workpiece moved forward under the pressure of guide wheel and friction force produced by the rotational ECAE punch groove wall. The workpiece continuously moved into the ECAE concave die under the 
friction force of ECAE punch A and ECAE punch B, when the workpiece arrived at the abutment, the metal flow direction changed 90 degrees angle and flows through the die hole, the continuous ECAE process is realized

With the continuous rotation of ECAE punch A, the severe shear deformation occurs at the die corner and the workpiece was continuously extruded from one side of the ECAE cavity die. The continuous ECAE forming of aluminum alloy was achieved, the deformation aging temperature can be controlled by using the cooling system with the machine, It is expected to achieve a dynamic continuous ECAE age forming. This is different from the coshearing ECAE, both work roll frictions and belt frictions are the driving force of coshearing ECAE forming. The continuous ECAE aging forming equipment designed by our group, the frictions between workpiece and ECAE punch A groove bottom, as well as the frictions between workpiece and the side wall are the driving force. ECAE punch B stationary punched. The frictions between workpiece and ECAE punch B are resistance; the forming process is very complex. The geometry of the die and the properties of tool and workpiece are key factors to realization of continuous ECAE forming, so the effect of die contours and properties of sample on internal stress-strain and temperature distribution should be clearly understood, that an optimum continuous dynamic ECAE process to produce electric conductive can be obtained.

The rigid viscoplastic finite element model of continuous ECAE forming process was firstly established, and then the process of $\mathrm{Al}-\mathrm{Zr}$ resistant heat alloy produced by continuous ECAE was simulated by using DEFORM-3D. The effective stress-strain and the temperature distribution of the continuous ECAE forming process were analyzed. The foundation for further research in the future was laid out.

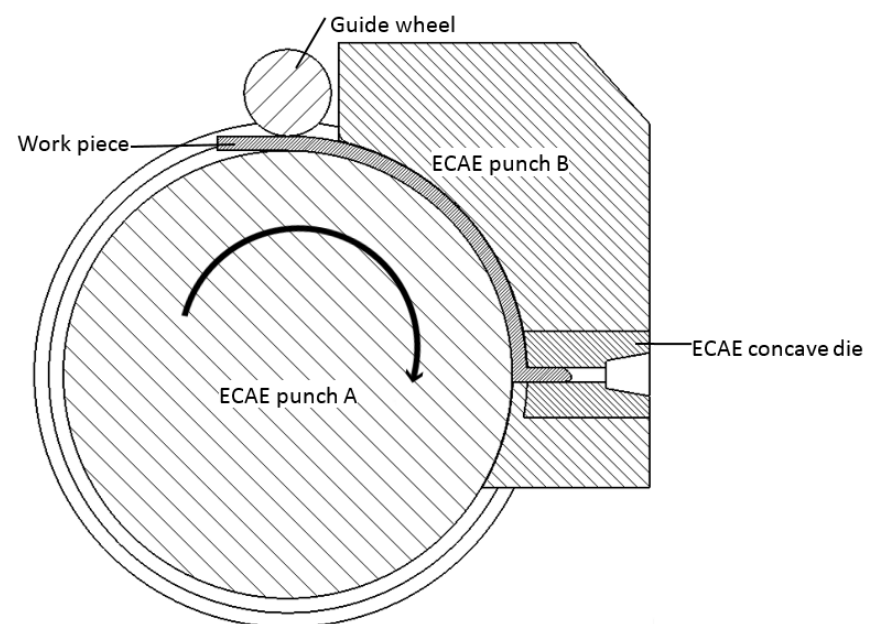

Fig.1 Continuous ECAE principle diagram

\section{The Establishment of Finite Element Model}

Currently, the optimal geometrical and processing parameters are usually obtained through experimental study or the numerical simulation method. Although experimental study is usually necessary in this investigation, it is difficult to get the material flow law and interior field variable distributions with much money and time cost by experimental study. Being finite element based numerical simulation is an effective tool to predict the material flow properties and interior field variables, so the metal continuous ECAE process is studied by using numerical simulation.

The continuous ECAE process is simulates by using the commercial metal forming finite element software DEFORM-3D in this study. The deformation behavior will be discovered.

Using the Levy-Mises rate equation, the Mises yield criterion and Markov variational principle the rigid viscoplastic finite element simulation (RVFEM) model is established. The RVFEM is suitable for forging, extrusion and rolling analysis of plastic bulk forming problems, and the deformation and heat transfer coupling process can be analysis.

In the simulation process, the material should obey the following assumptions. Neglect the elastic 
deformation of the material and inertia, Material is homogeneous and isotropic, The volume of material constant; The material obeys the Mises yield criterion; The constitutive relationship is Levy-Mises equation; Rigid plastic material only has strain hardening, and rigid visco plastic material has the strain hardening and strain rate hardening.

Due to the real deformation of material is very complex, the above assumptions according to the basic hypothesis of rigid viscoplastic theory is needed. meanwhile, the structure of screw hole and chamfering etc. have no effect on metal forming, these structure should be neglected and only retain the structure have effect on the metal forming. Assume the production is stable and each parameter tends to be stable. The model is established as follow, workpiece was extruded a certain length from the die, every parts drawn and assemble them in the 3D CAD software SolidWorks with exporting STL file, and then put it into the DEFORM-3D simulation pre-processer. Taking the workpiece as the plastic body, and set the tool as the rigid body and the temperature of the workpiece during continuous ECAE not isothermal. Due to the workpiece and the die structure in the selected model is symmetrical, and the workpiece do not flow in the normal direction of the symmetric surface, its stress conditions and load constraints is also symmetrical, therefore, select a half integral model as the research object. After importing entity need to set the material of workpiece and die.The material of workpiece is $\mathrm{Al}$ - $\mathrm{Zr}$ heat-resistant alloy, measured by the test, the yield stress is $83.6 \mathrm{MPa}$, the young's modulus is $69 \mathrm{GPa}$, Poisson's ratio is 0.33 , the coefficient of thermal conductivity is $230 \mathrm{~W} / \mathrm{m}$ - K. ECAE punch and the guide wheel add rotate load by the opposite direction. The rest parts are fixed. The workpiece use tetrahedron mesh, the initial cell number is 39977, a continuous ECAE finite element mesh model is built. As shown in Fig. 2.

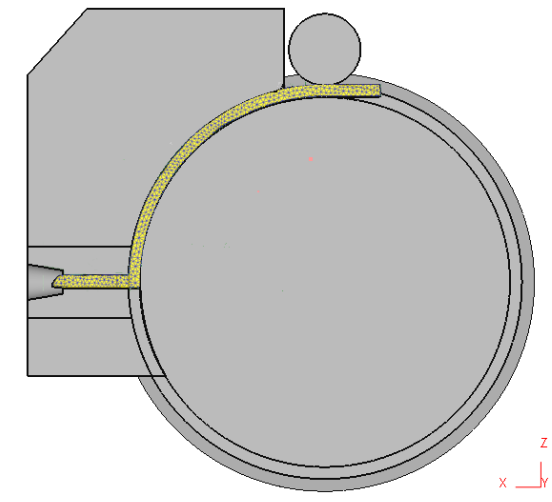

Fig.2 Continuous ECAE finite clement mesh model

\section{The Setting of Simulation Parameters}

According to in the previous literature settings, and simulate the optimum parameters are selected as Tab. 1.

Tab.1 Friction coefficient used for simulation

\begin{tabular}{cccc}
\hline Workpiece and punch A & $\begin{array}{c}\text { Wokpiece and guide } \\
\text { wheel }\end{array}$ & Workpiece and punch B & $\begin{array}{c}\text { Workpiece and } \\
\text { concave die }\end{array}$ \\
0.25 & 0.25 & 0.1 & 0.2
\end{tabular}

Initial temperature of Workpiece, guide wheel punch B and punch A concave die are $220^{\circ} \mathrm{C}$, The rotational speed of punch A and the guide wheel is 1.0472 RPS and -5.7595 RPS respectively.

\section{Results and Discussion}

\section{The Stress and Strain Distribution of the Continuous Dynamic ECAE Forming Process}

The stress distribution is shown in the Fig. 3. It can be seen that the stress distribution of deformed metal existing in the cavity from the beginning to the exit is inhomogeneous. The stress 
gradually increase with the deformed metals moving forward to the export, the stress reached the maximum at the turn angle regions. After that the stress decreases with the workpiece forward to the die exit. the stress gradually becomes more homogeneous. However, the stress near the center along the radius is bigger than that of faraway. The equivalent stress reaches the maximum value of $141 \mathrm{MPa}$ in the bottom of the die corner. And its distribution in the die cavity and is homogeneous and with the workpiece is extruded out the die cavity, the equivalent stress decreases decrease to zero after die-out. The strain distribution is similar to the stress distribution. The equivalent strain reaches the maximum with the value of 29.2 in the bottom of the die corner as shown in Fig. 4,

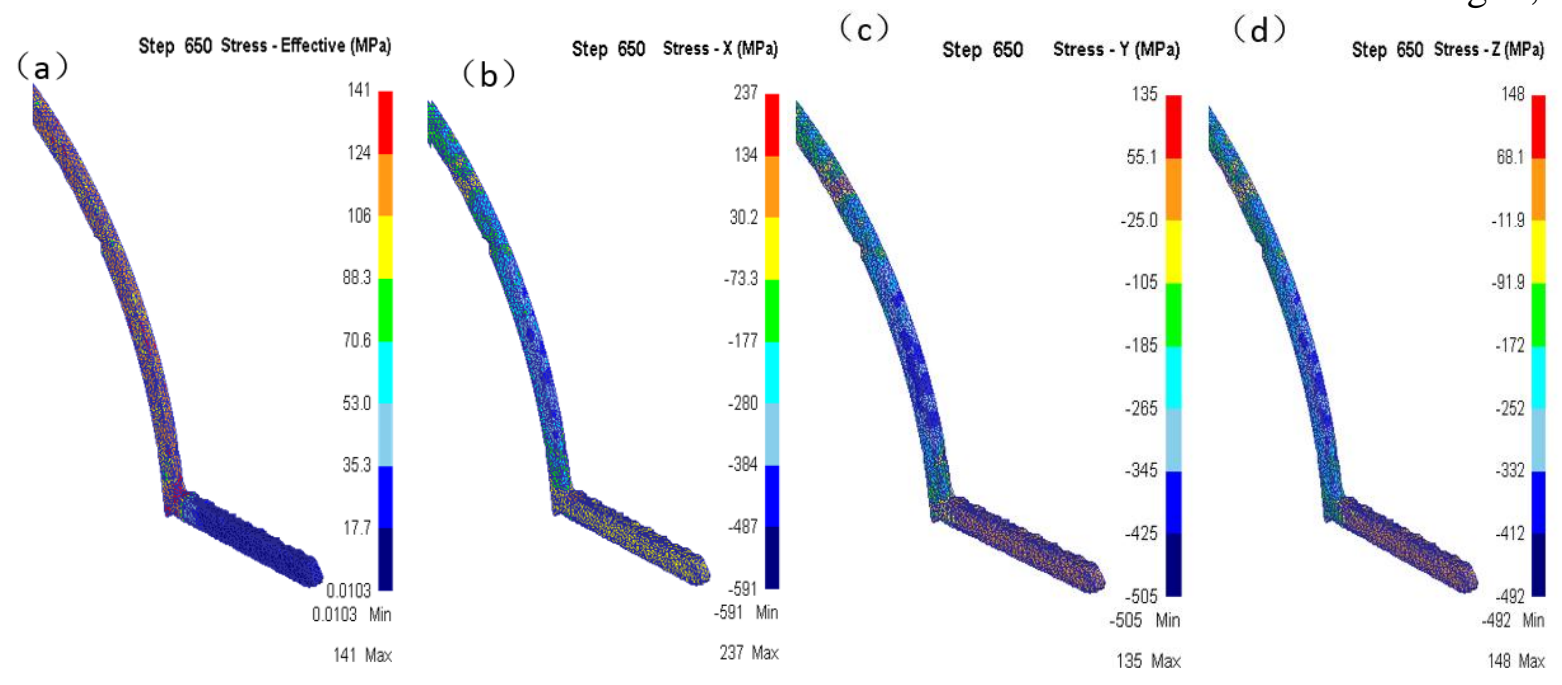

(a)- Effective stress; (b)-stress-X; (c)-stress-Y; (d)-stress-Z

Fig. 3 Stress distribution of the continuous dynamic ECAE forming process

The equivalent strain and each direction strain distribution tend to be homogeneous after the sample is extruded out. The reason is that there is speed difference between ECAE punch A and ECAE punch B. ECAE punch A with cavity is rotated by power motor, ECAE punch B is standstill (seeing in the Fig.1). The metals contacting to the punch $\mathrm{A}$ is undertaken more friction force, especially to the bottom of slot of ECAE punch A, so these metals near to the bottom of slot as well as the side wall of the punch $\mathrm{A}$ is flow faster than that of near punch $\mathrm{B}$, so the stress near the radius center is bigger than far of that. With the metals moving to the die turn angle area, the accumulated force becomes bigger, so the stress increases. The effective strain reaches the maximum value of 29.2 in the bottom of the die corner as shown in Fig. 4. The effective strain and each direction strain distribution tend to be homogeneous after the workpiece is extruded out. the reason is that the deformation of metals exiting in cavity becomes larger from the beginning to die-out along the hoop of ECAE forming.

(a)

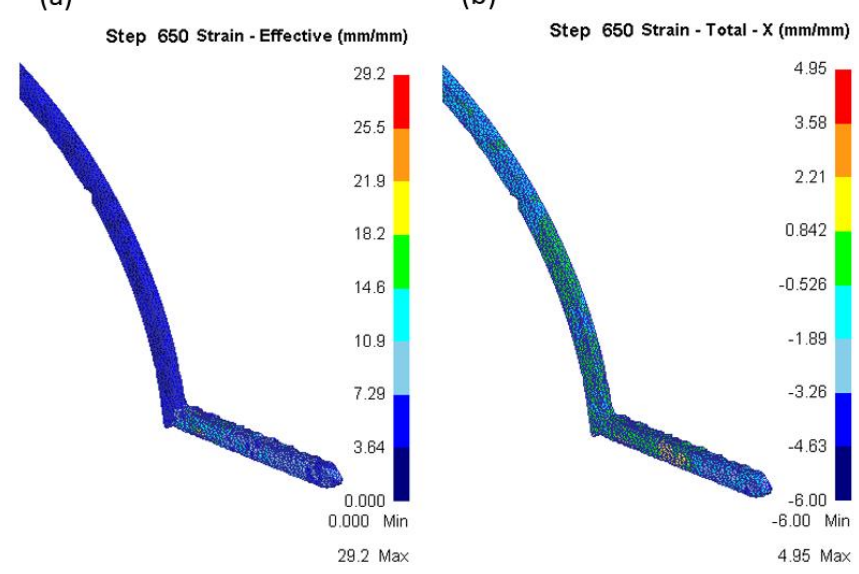

(c)

Step 650 Strain - Total - Y $($ mmm (d) Step 650 Strain - Total - Z (mmmm)

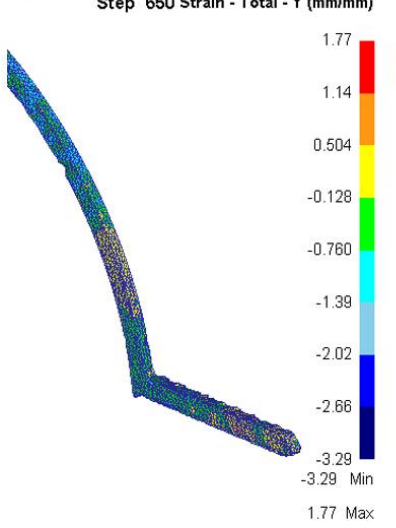

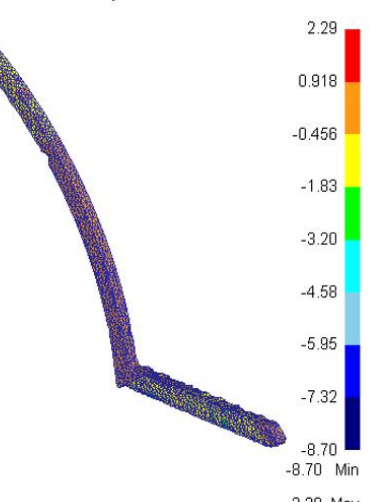

(a)-Effective strain; (b)-strain-X ; (c)-strain-Y; (d)-strain-Z

Fig.4 Strain distribution of the continuous dynamic ECAE forming process 


\section{Temperature Distribution of the Continuous Dynamic ECAE Forming Process}

The temperature distribution of continuous dynamic ECAE process is shown in Fig. 4. The temperature of the deformed metals increase from the metals entering the cavity formed by punch A and punch B to ECAE cavity die, the temperature reach the top in the die entrance corner area. Then the temperature decrease with the workpiece extruded out. During the continuous extrusion process, it can be seen that the entrance temperature of the workpiece is about $200^{\circ} \mathrm{C}$, the maximum temperature in the die entrance corner $300^{\circ} \mathrm{C}$ and the die-out temperature $250^{\circ} \mathrm{C}$. The result is coaction supported by the deformation heat and friction, because the deformation and friction force of metals existing in the cavity from entrance to ECAE die gradually increase, the temperature of sample existing die -corner zone is high of $300^{\circ} \mathrm{C}$, This temperature is fit for dynamic forming.

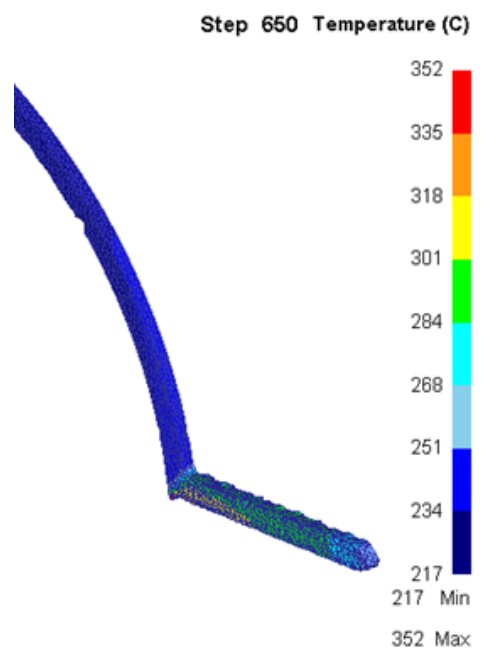

Fig. 5 Temperature distribution of the continuous dynamic ECAE forming process

\section{Conclusion}

(1) Continuous dynamic ECAE forming can be successfully developed by using reasonable die geometry and appropriate parameters.

(2) The equivalent stress-strain increase from the sample entrance the ECAE cavity to die turn angle zone, reaches maximum stress and strain in the bottom of the die corner is 141Mpa and 29.2 respectively. The temperature of the workpiece increased gradually during the forming process. The maximum temperature is $300^{\circ} \mathrm{C}$, homogeneous parts man be prepared by using multi EACE process like traditions.

\section{Acknowledgement}

This research is financially supported by National Natural Science Foundation (51174139).

\section{References}

[1]S. $\mathrm{Xu}, \mathrm{G}$. Zhao, X. Ren, et al. Numerical investigation of aluminum deformation behavior in three-dimensional continuous confined strip shearing process, J. Materials Science and Engineering: A , 476(1-2) (2008)281-289.

[2]W. Wei, W. Zhang, K. X. Wei, et al. Finite element analysis of deformation behavior in continuous ECAP process, J. Materials Science and Engineering: A, 516 (1-2)(2009)111-118.

[3]V.M. Segal. Equal channel angular extrusion of flat products, J. Materials Science and Engineering: A, 476(1-2) (2008)178-185.

[4]V. M. Segal. Mechanics of continuous equal-channel angular extrusion, J. Journal of Materials 
Processing Technology, , 210(3)(2010)542-549.

[5]T. Sakai, H. Miura, A. Goloborodko, et al. Continuous dynamic recrystallization during the transient severe deformation of aluminum alloy 747, J. Acta Materialia, 57(1) (2009)153-162.

[6]G. J. Raab, R. Z. Valiev, T. C. Lowe, et al. Continuous processing of ultrafine grained Al by ECAP-Conform, J. Materials Science and Engineering: A, 382(1-2)(2004)30-34.

[7]. H. Utsunomiya, K. Hatsuda, T. Sakai, et al. Continuous grain refinement of aluminum strip by conshearing, J. Materials Science and Engineering A, 372(1-2) (2004)199-206.

[8]H. J. Roven, M. Liu, J. C. Werenskiold. Dynamic precipitation during severe plastic deformation of an Al-Mg-Si aluminum alloy, J. Materials Science and Engineering A, 483-84(SI) (2008)54-58.

[9]R. Kaibyshev, K. Shipilova, F. Musin, et al. Continuous dynamic recrystallization in an Al- $\mathrm{Li}-\mathrm{Mg}-\mathrm{Sc}$ alloy during equal-channel angular extrusion, J. Materials Science and Engineering A, 396(1-2) (2005)341-351. 